\title{
A Recipe for Universal Vaccines
}

\author{
Researchers use nonequilibrium statistical physics methods to guide the \\ design of vaccines that are effective against many strains of a virus, a holy \\ grail of immunology.
}

By Katherine Wright

$\int$ ust over a year ago, physicists around the world shifted their research efforts to the field of infectious diseases to help combat the COVID-19 pandemic. In the US, soft-matter physicists trialed a technique for detecting SARS-CoV-2, the virus that causes COVID-19; in Switzerland, high-energy physicists tested an inexpensive ventilator technology that could help sufferers breathe; and in the UK, condensed-matter physicists donated their personal and protective equipment to the country's hospitals.

Applying the tools of physics to infectious diseases is an old idea, however, as physicists have worked for decades on understanding how diseases spread and how viruses evolve. Arup Chakraborty of the Massachusetts Institute of Technology is one such physicist. He and his postdoc Raman Ganti now present results of their recent project to use

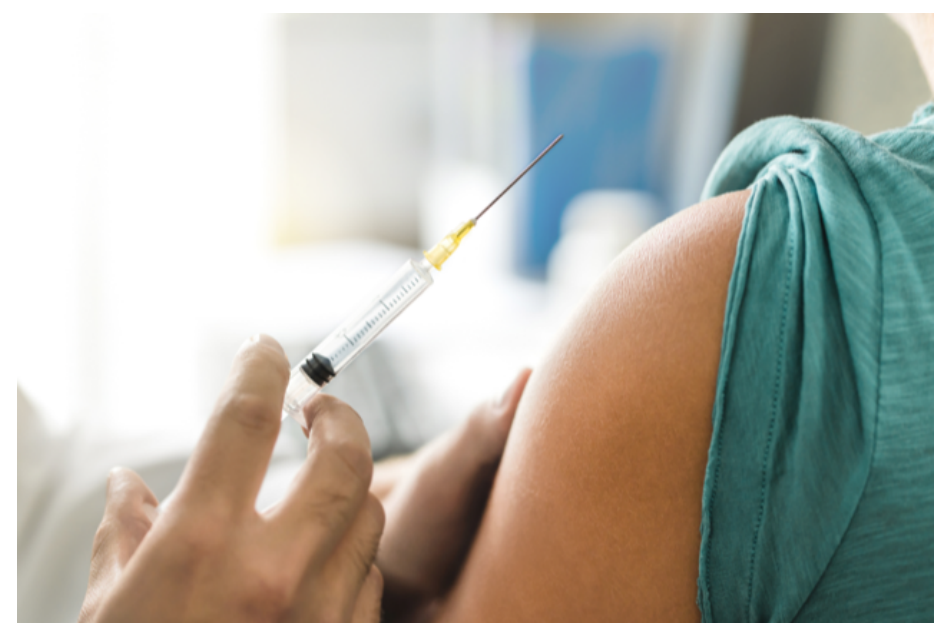

Vaccines might be optimized by choosing a sequence of different antigens for the initial and booster shots.

Credit: terovesalainen/stock.adobe.com statistical-mechanics methods to predict the characteristics of the optimal antigens for "universal" vaccines for rapidly mutating viruses, such as influenza [1]. Their insights should also be applicable to creating broad-coverage vaccines for a range of viruses from HIV to SARS-CoV-2.

"Vaccinations have saved more lives than any other medical procedure," Chakraborty says. "Nonetheless, effective vaccines do not exist for highly mutable pathogens-that's why we need a seasonal flu shot. Now that COVID-19 is mutating, we could end up needing repeated vaccinations for that virus too."

Each year, medical professionals inject hundreds of millions of flu vaccines into the arms, legs, and bottoms of people around the world. These vaccines contain an antigen from a weakened or inactivated strain of the flu virus that stimulates the body to produce antibodies. These antibodies target so-called spike proteins that protrude from the viruses and that are used by the viruses to enter cells.

Different antibodies target different regions on the spike protein, so the body goes through an evolutionary process-involving so-called B cells-to select which antibodies to develop. The most commonly selected flu virus antibodies target the regions of spike proteins that rapidly mutate. That means that once a new strain of flu develops, the virus can evade the body's defenses, and a new vaccine needs to be created. There are, however, regions of spike proteins that rarely vary.

Immunologists would like to create a vaccine that triggers the production of antibodies that target these unchanging regions. These antibodies, which are known as broadly neutralizing antibodies, have been isolated in some people who are 
naturally infected with influenza or HIV, but they occur rarely and are not seen in those given vaccines, Chakraborty says. "The question is, how can we vaccinate in such a way that these rare antibodies become the dominant ones?"

To address that question, Chakraborty and Ganti turned to methods from nonequilibrium statistical physics, creating a model that predicts the type of antibody produced. They chose a two-vaccination protocol, a common strategy in which an initial shot is followed shortly by a booster shot. In the model, each of the vaccines contains a different composition of virus antigens-basically different strains of the inactivated pathogen. The body's B cells respond to the antigens by rearranging their population in an evolutionary process that resembles a perturbed system reaching equilibrium. The model controls how perturbing each antigen is, based on how different it is from the antigens that the B cells already "know" from previous exposures.

To interpret the results, the duo applied a statistics-based learning theory, widely used in education research and machine learning. "Developing antibodies is a learning process for the body, so we thought the theory could provide interesting insights," Ganti says.

In the context of this theory, the duo found that broadly neutralizing antibodies can dominate under certain learning conditions. First, the antigen in the initial vaccine mustn't be too similar or too different from ones the body has already encountered. In other words, the level of learning difficulty for the B cells can't be too easy or too hard, otherwise the final antibodies that the cells develop won't be broadly neutralizing.
"Getting the antigen right in the first vaccination is critical," Ganti says. Second, the learning level must be higher for the booster shot than for the initial one. (Again, there is a sweet spot for how high.)

When those learning steps are correctly optimized, the model indicates that broadly neutralizing antibodies will dominate. "The results show that if we get the learning right, we can steer antibody development, so that an otherwise rare evolutionary trajectory becomes much more likely," Chakraborti says. The duo is now working on translating this learning strategy into an optimal genetic sequence that researchers working on universal vaccines can use to pick specific antigens for the two vaccinations.

Arvind Murugan of the University of Chicago says that the new results "suggest a more systematic and principled way of figuring out optimal vaccination protocols." Murugan is particularly taken by the use of learning theory in the study. "Fundamentally, evolving a broadly neutralizing antibody is sort of like learning the right lessons from your history: you want to focus on aspects of your past experience that will be relevant for future challenges, rather than idiosyncratic details that'll never be relevant again," he says.

Katherine Wright is the Deputy Editor of Physics.

\section{REFERENCES}

1. R. S. Ganti and A. K. Chakraborty, "Mechanisms underlying vaccination protocols that may optimally elicit broadly neutralizing antibodies against highly mutable pathogens," Phys. Rev. E 103, 052408 (2021). 